\title{
Visualization for Genetic Evolution of Target Movement in Battle Fields
}

\author{
S. Baik ${ }^{1}$, J. Bala ${ }^{2}$, A. Hadjarian ${ }^{3}$, P. Pachowicz ${ }^{3}$, J. Cho ${ }^{1}$, and S. Moon ${ }^{1}$ \\ ${ }^{1}$ College of Electronics and Information Engineering, Sejong University, \\ Seoul 143-747, Korea \\ \{sbaik, jscho, sbmoon\} @sejong.ac.kr \\ ${ }^{2}$ School of Information Technology and Engineering, \\ George Mason University, \\ Fairfax, VA 22030, U.S.A \\ jbala@gmu . edu \\ ${ }^{3}$ Sigma Systems Research, Inc. \\ Fairfax, VA 22032, U.S.A \\ \{ahadjarian, ppach\}@sigma-sys.com
}

\begin{abstract}
This paper focuses on the development of an interactive 3D visualization environment for displaying predicted movements by genetic evolution. The integration of interactive 3D visualization and the genetic search approach can relieve the burden of image exploitation tasks occurring due to an explosion of imagery data obtained in battle fields. The visualization component was implemented using In3D - a cross-platform Java class library designed to enable the creation of interactive $3 \mathrm{D}$ visualizations of complex information spaces.
\end{abstract}

\section{Introduction}

Recently, military missions have expanded into new and uncharted territories such as coalition operations; highly mobile operations; enhanced C4I (Command, Control, Communications, Computers and Intelligence); peacekeeping and humanitarian operations; law enforcement operations of counter-narcotics and counterterrorism; monitoring treaties and weapons proliferation along with further "operations other than war." These increased areas of responsibility bring with them a greater need for imagery support. In this context, this explosion of available imagery data overwhelms the imagery analysts and outpaces their ability to analyze it. Consequently, the analysis tasks becoming bottlenecked in the imagery community. This situation generates an urgent need for new techniques and tools that can assist analysts in the transformation of this huge amount of data into a useful, operational, and tactical knowledge. The approach presented in this paper applies Genetic Algorithms (GAs) learning techniques to evolve new individuals in the population of movements in order to converge the evolution process toward optimal (most probable) movements. The major innovations in this approach are graphically depicted in Fig. 1. 


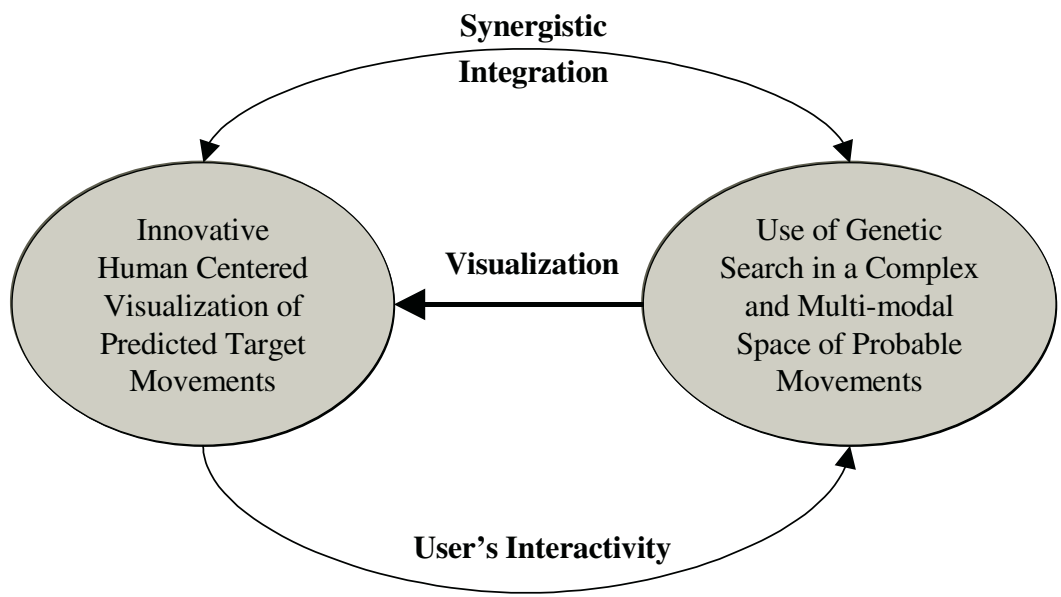

Fig. 1. Innovative concepts of Genetic Evolution Movement

\section{Genetic Evolution of Target Movement}

In a previous work [1], we focused on the genetic evolution approach for target movement prediction. Genetic Algorithms (GAs) [2,3] are used to generate the population of movement generation operators. The population evolves by using crossover and mutation and only the strongest elements survive, thus contributing to improved performance in terms of more probable/optimal movements. This contribution is used as an objective evaluation function to drive the generation process in its search for new and useful movement generation operators. When compared to the other researches ${ }^{1}$, the main differentiators can be summarized in the following two points: 1 ) GAs use payoff (objective function) information, not derivatives or other auxiliary knowledge. Other search techniques require much auxiliary information in order to work properly; and 2) GAs use probabilistic transition rules, not deterministic rules. GAs use random choice as the tool to guide a search towards regions of the search space with likely improvement (i.e., more probable movements).

\section{Interactive 3-D Visualization for Target Movement Prediction}

It becomes more popular to use visualization techniques [4-6] to facilitate the development of GA systems. We focused on the development of an interactive 3D visualization environment for displaying predicted movements by genetic evolution. The system-interfacing component and visualization component was implemented using

${ }^{1}$ BONN Corp. has developed an enhancement to an existing movement prediction system, called the Tactical Movement Analyzer (TMA). The NATO Reference Mobility Model (NRMM) is the Army's accredited mobility performance prediction model. 
In $3 \mathrm{D}^{2}$ - a cross-platform Java class library designed to enable the creation of interactive 3D visualizations of complex information spaces. The use of this library is pivotal in the implementation of the visualization environment for the genetic evolution of movements.

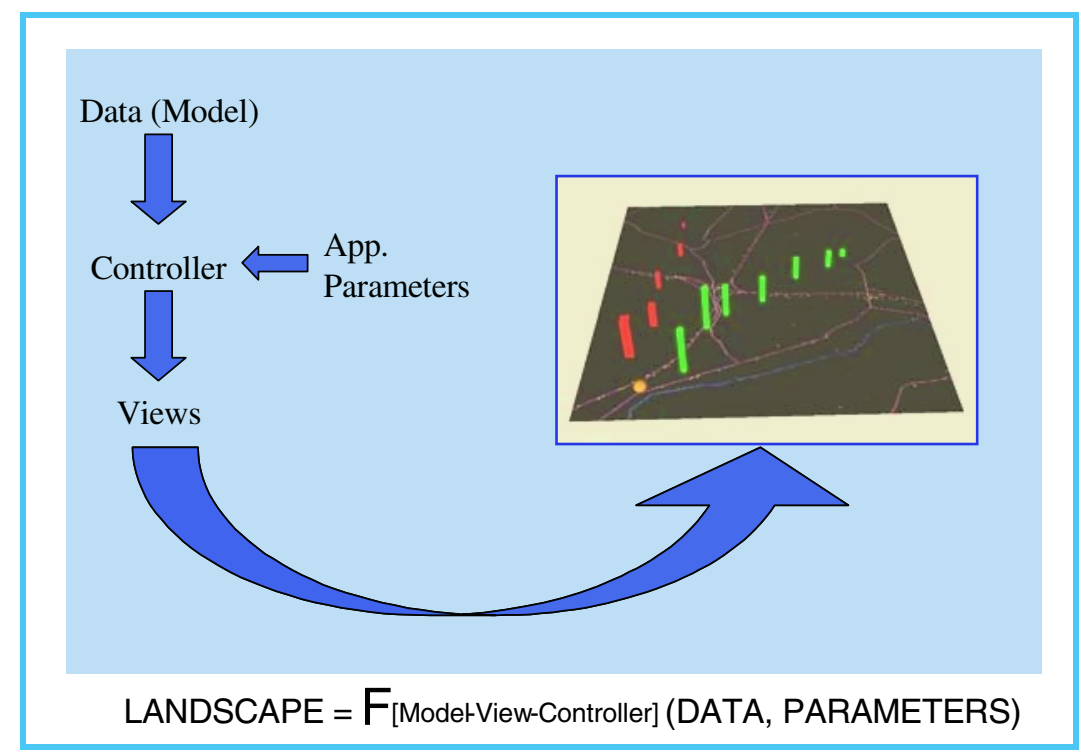

Fig. 2. A Model-View-Controller display paradigm

The visualization component implements the Model-View-Controller paradigm of separating imagery data (model) from its visual presentation (view). Interface elements (Controllers) act upon models, changing their values and effectively changing the views (Fig. 2). The Model-View-Controller paradigm supports the creation of applications which can attach multiple, simultaneous views and controllers onto the same underlying model. Thus, a single landscape (imagery and objects) can be represented in several different ways and modified by different parts of an application. The controller can achieve this transformation with a broad variety of actions, including filtering and multi-resolution, zooming, translation, and rotation. The component provides navigational aids that enhance users' explorative capabilities (e.g., the view from above provides a good overview of the information but it is not until zooming in and around and inspecting small items that the user gets a detailed understanding). Fig. 3 depicts an example of the prediction landscape.

\footnotetext{
${ }^{2}$ It was developed by Visual Insights Corp.
} 


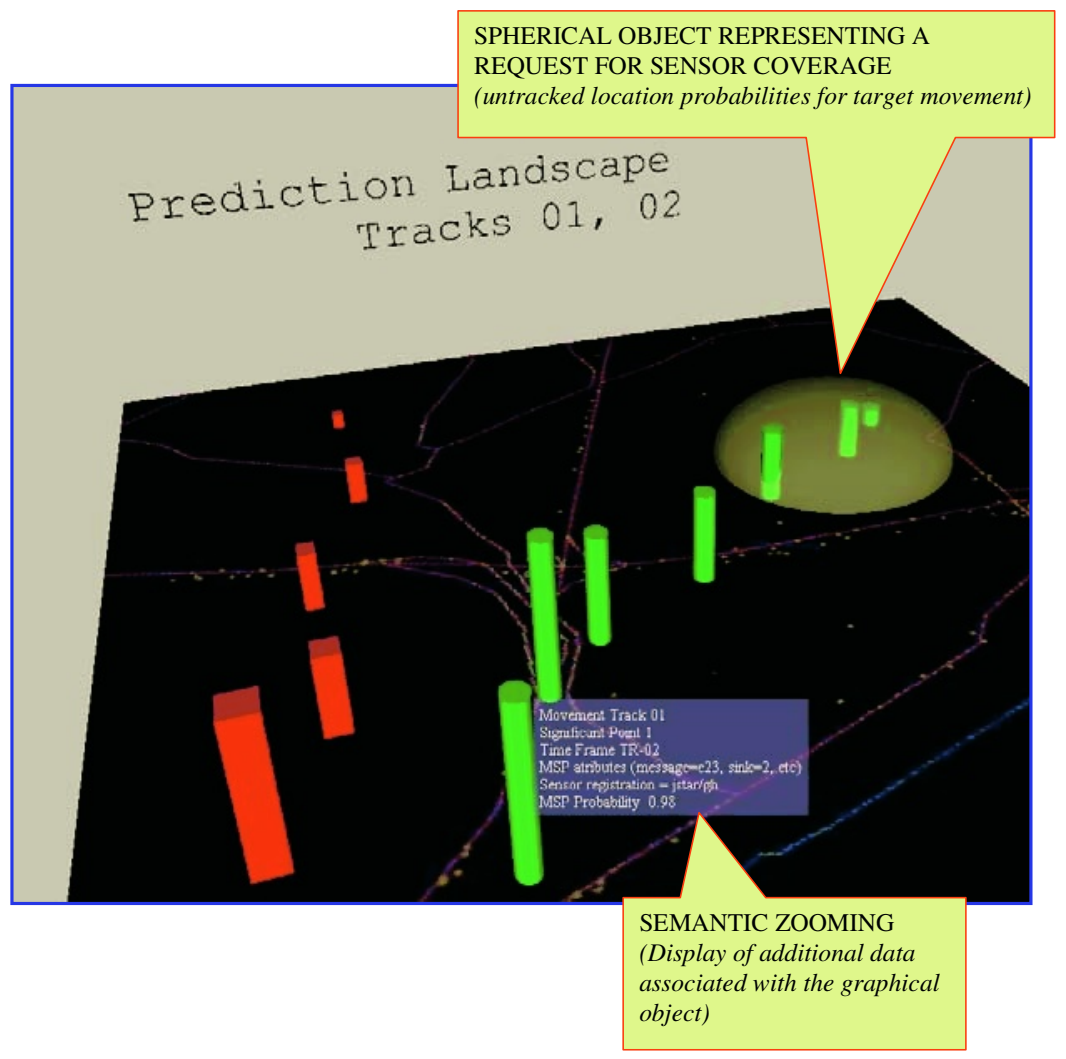

Fig. 3. Prediction landscape for Genetic Evolution of Movements

\section{References}

1. S. W. Baik, J. Bala, A. Hadjarian, and P. Pachowicz: Genetic Evolution Approach for Target Movement Prediction, Lecture Notes in Computer Science Vol. 3037, 2004

2. A. Brindle: Genetic algorithms for function optimization, Ph.D. Thesis, Computer Science Dept., Univ. of Alberta, 1981

3. K. A. DeJong: Adaptive system design: a genetic approach, IEEE Trans. Syst, Man, and Cyber, Vol. SMC-10, No. 9, pp. 566-574, Sept. 1980

4. W. B. Shine and C. F. Eick: Visualizing the evolution of genetic algorithm search processes, IEEE International Conference on Evolutionary Computation, pp. 367-372, April 1977

5. E. Hart and P. Ross: GAVEL - A New Tool for Genetic Algorithm Visualization, IEEE Trans on Evolutionary Computation, Vol. 5, No. 4, pp. 335-348, August 2001

6. A. M. Malkawi, R. S. Srinivasan, Y. K. Yi and R. Choudhary: Decision Support and design evolution: integrating genetic algorithms, CFD and visualization, Automation in Construction, Vol. 14, pp. 33-44, 2005 